\title{
Cardiovascular Mortality: An overview of Premature Death in the Republic of Moldova
}

\author{
Elena Raevschi ${ }^{*}$ \\ ${ }^{1}$ University of Medicine and Pharmacy "Nicolae Testemitanu, \\ Chisinau, 2004, Republic of Moldova. \\ *elena.raevschi@usmf.md
}

\begin{abstract}
YPLL health outcome indicator (Years Potential Life Lost) proposed in the Global Burden of Disease 1990 study [1] to estimate the burden of disease in a population, shifts the emphasis from more familiar health measure of occurrence of death to quantify the burden of loss. YPLL allows selectively evaluate the mortality in younger age groups. The purpose of research is to estimate the impact of premature death caused by major contribution cardiovascular diseases in the Republic of Moldova. This research is a population descriptive study. The study estimated that the population of working age (up to 62 years) in Moldova lays $70 \%$ of the major contribution cardiovascular diseases YPLL average (y.2008-2012). Systematic evaluation of YPLL health outcome indicator leads to increase priority on community interventions and evidence-based planning of cardiovascular risk management in the Republic of Moldova.
\end{abstract}

Keywords: Cardiovascular disease, premature death, YPLL, cardiovascular risk management planning

\section{Introduction}

Cardiovascular diseases are known worldwide as the ,top killer of the $21 \mathrm{st}$ century", having a mortality level higher than all combined cancer diseases. World Health Organizations' (WHO) data show that cardiovascular diseases is the highest mortality cause in the last decade $(31 \%$ of total decease cases in 2010 year) [2].

Cardiovascular mortality rate in Republic of Moldova exceeds the global average value by two times for at least a decade by now [3].

WHO prognosis for the upcoming 2020 and 2030 years aren't so bright, the number of mortality cases will reach up to 20 million cases in year 2020 and 24 million in year 2030. WHO calculated that cardiovascular diseases, hearts attacks and diabetes annually reduce a countries' GDP by $1-5 \%$ [4].

According to WHO strategies, premature mortalities caused by non-communicable chronic diseases can be reduced by $25 \%$ until year 2025 , by achieving this goal we will have a simultaneous reduction of premature cardiovascular mortality by correcting behavioral risk factors among the population [5]. While international statistics are using potential health outcome indicators like YPLL and DALY, which were proposed by the WHO for assessment of disease burden, in Republic of Moldova systematic assessment of populations' health status is calculated, most times, by analyzing crude mortality that are centered on each occurrence, rather than the caused losses.

\section{Study Design}

\section{The aim of the study}

Evaluation of mortality impact caused by cardiovascular diseases in Republic of Moldova. The evaluation is based on the burden of loss caused by premature decease to improve cardiovascular risk management planning.

\section{Material and Methods}

In order to achieve the aim was applied a descriptive method of investigation using 
the data provided by the World Health Organization (2010-2014) and National Center of Health Management from the Republic of Moldova (2014) [6].

Research design covered three classic directions of descriptive study investigation: 1 - by time, 2 - by place and 3 - by personal characteristics. Data analysis by time included the period 2008-2012, depending on place - the Republic of Moldova, rural and urban areas and depending on the person - by sex and age.

Years of Potential Life Lost (YPLL) was calculated using the equation (1):

$$
Y P L L=\sum_{i=1}^{11}\left(70-a_{i}\right) \times d_{i}
$$

YPLL - years of potential life lost $i$ - number of groups of quinquennial age; 70 - superior limit of age that is considered a premature decease; $a_{i}$ - age class center; $d_{i}$ - number of deceases in each age group.

\section{Results}

Populations' statistical indicator of mortality is considered to be among the most important ones that influences life expectancy and is an undesirable outcome of morbidity and disability, also including quality of life.

\section{Major contribution cardiovascular diseases for mortality}

Mortality by circulatory diseases among the adult population of Republic of Moldova in the period 2008-2012 year and by cardiovascular diseases of major contribution for the same period demonstrated a decrease from 657,4 (y. 2008 ) to 641,6 (y. 2012) per 100,000 population and 635,4 (y. 2008) to 619,3 (y. 2012) per 100,000, respectively.

With this, it is important to mention the unstable character of the decreasing trend represented above. Therefore, for years 2008-2010 took place an increasing of the mortality level of circulatory diseases from 657,4 (y. 2008) to 663,2 (y. 2009) followed by a frequency hop with a level of 688,1 for each 100,000 population (y. 2010). Subsequently was produced a rapid decrease in year 2011, reaching a level of 633,4 per 100,000 , which was again followed by an increase up to 641,6 per 100,000 population.

Mortality rate by major contribution cardiovascular diseases for years 2008-2010 demonstrated a value increase from 635,4 (y. 2008) to 641,9 (y. 2009) followed by a frequency hop of 662,4 per 100,000 population for year 2010. Subsequently was produced a decrease in year 2011, down to 612,4 per 100,000 population, which was again followed by an increase up to 619,3 per 100,000. Therefore, major contribution cardiovascular diseases for mortality in sum manifested the same logistic of changes described in the circulatory diseases case.

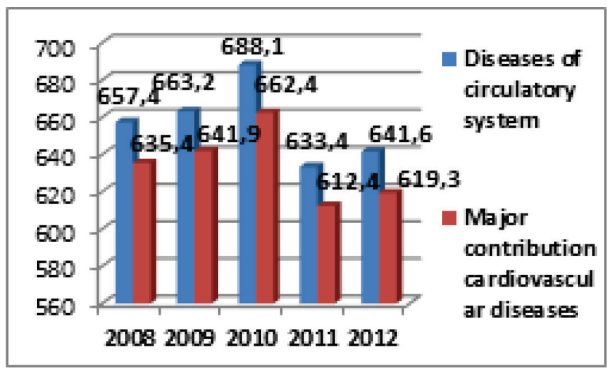

Figure 1. Mortality rates by circulatory system diseases and major contribution cardiovascular diseases, Republic of Moldova, 2008-2012 (per 100,000).

Analysis of major contribution cardiovascular diseases in dependence of clinical form (ischemic heart disease, cerebrovascular disease and hypertensive disease) showed that only ischemic heart disease is maintained the stated tendency. Therefore, for years 2008-2010 was seen an increase of mortality rate by ischemic heart disease from 425,3 (y. 2008) to 438,8 (y. 2009) followed by a frequency hop of 465,07 per 100,000 population for year 2010. Subsequently was produced a remarkable decrease in year 2011, reaching a level of 419,27 per 100,000 population, which was again followed by a small increase up to 420,2 per 100,000 population. In cerebrovascular disease case 
for years 2008-2012 was not seen a significant frequency hop, manifesting in this way a much more stable decrease tendency than major contribution cardiovascular diseases and ischemic heart disease. In this context, rate for cerebrovascular disease for years 20082011 manifested a stable decrease from 189,9 (y.2008) down to 168,15 (y.2011) per 100,000 population, followed by a modest increase up to 172,07 per 100,000 population in year 2012 "Figure 2".

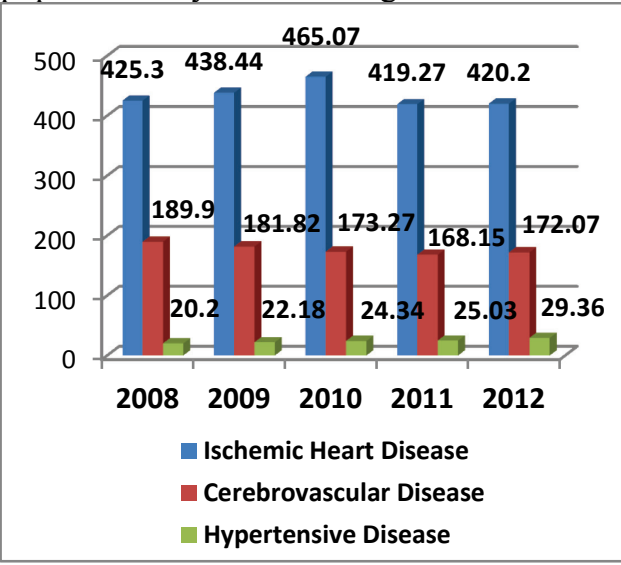

Figure 2. Mortality rates by major contribution cardiovascular diseases, Republic of Moldova, 2008-2012 (per 100,000).

At the same time, for the same research period hypertensive disease manifested a continuous trend of value increase from 20,2 to 29,36 per 100,000 population.

\section{Aspects of premature death by major cardiovascular diseases}

In the distribution of the phenomenon due to premature deceases, cause by major contribution cardiovascular diseases in dependence of groups of quinquennial age, was determined that the greatest proportion had age group 55-59 years $(25,87 \%)$, followed by age $60-64$ years $(20,18 \%)$ and $50-54$ years $(18,64 \%)$. It is important to note that all three groups represent period of working age (economically active population). Additionally, in $18,56 \%$ of total years of potential life lost because of major cardiovascular diseases are assigned to the age groups that cover a period of 4050 years and groups of a younger age (less than 40 years) in $6.87 \%$ of cases.

Unlike major cardiovascular diseases and hypertensive disease, premature decease phenomenon distribution, caused by ischemic heart disease and cerebrovascular disease, showed an inclination towards younger aged groups "Figure 3".

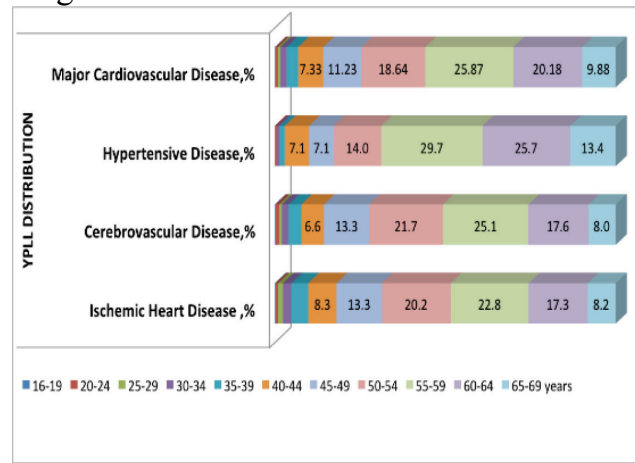

Figure 3. Years of Potential Life Lost distribution under the age of 70, Republic of Moldova, 2008-2012 (\%).

Therefore, in cerebrovascular diseases' distribution the highest determined value presented age group of 55-59 years $(25,1$ $\%)$, followed by age $50-54$ years $(21,7 \%)$ and afterwards by age $60-64$ years $(17,6 \%)$. Age group of 40-50 years demonstrated $19,9 \%$ and younger groups ( $<40$ years) of $7,7 \%$ from the total years of potential life lost due to hypertensive disease.

Ischemic heart disease shows the highest value of premature decease in age groups $55-59$ years $(22,8 \%)$, followed by age group $50-54$ years $(20,2 \%)$ and afterwards by age group 60-64 years $(17,3 \%)$. Age group of $40-50$ years showed a percentage of $21,6 \%$ and younger age groups ( $<40$ years) of 9,9 $\%$ from the total years of potential life lost due to ischemic heart disease „Figure 3”.

In the context, involvement in avoidable decease phenomenon due to major cardiovascular diseases among population of working age, effectuated analysis in the current study has estimated high values for economically active population. 
Therefore, estimated percentage of premature decease among working age population caused by ischemic heart disease is $74,5 \%$, cerebrovascular disease - $74,4 \%$ and hypertensive disease - $61 \%$ from the total years of potential life lost between the period 2008-2012 "Figure 4".

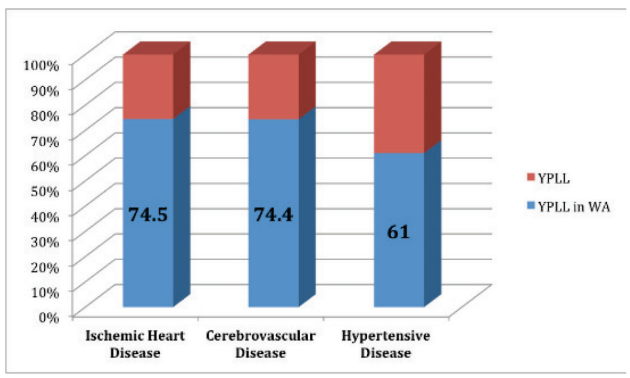

Figure 4. Proportion of Years of Potential Life Lost in working age (YPLL in WA) by major contribution cardiovascular disease population under the age of 70, Republic of Moldova, 2008-2012 (\%).

Evaluation of disease burden focused on losses produced by death demonstrated that in the period 2008-2012 was estimated an increase from 10.3 up to 10.5 of YPLL number average for a death caused by ischemic heart disease "Figure 5".

Therefore, achieving a general tendency of mortality level decrease due to ischemic heart disease among working age adult population was produced predominantly at the expense of advanced age groups.

The analysis of the average YPLL number for each occurred death due to cerebrovascular disease showed that in this case the tendency of decrease mentioned above for the period 2008-2012 was produced predominantly at the expense of advanced age groups, demonstrating an average YPLL of: $10.1 ; 10.1 ; 10.5 ; 9.9$; 10.1 for a occurred death "'Figure 5".

Increased level of mortality due to hypertensive disease among working age adult population between the periods of 2008-2012 was predominantly at the expense of age groups higher than 60 years (from 17.5 up to 32.1 per 100,000 population). Decrease of the average YPLL from 8.4 down to 7.5 for each occurred death due to hypertensive disease in the period of 2008-2012 demonstrates the same situation; tendency of decrease for the period 2008-2012 was produced predominantly at the expense of advanced age groups "Figure 5".

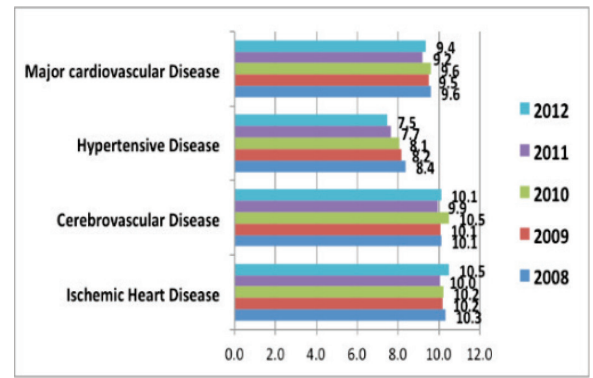

Figure 5. Years of Potential Life Lost distribution for one death under the age of 70 ,

Republic of Moldova, 2008-2012 (\%).

\section{Discusions}

The different approaches of YPLL measure (versus crude mortality) incorporating age at death can serve as an index of the social and economic consequence of mortality.

Using YPLL statistics in addition to more familiar crude mortality rates shifts the emphasis from occurrence of deaths to losses caused by premature mortality.

Unlike major cardiovascular diseases and hypertensive disease, the structure of premature decease phenomenon, caused by ischemic heart disease and cerebrovascular disease, estimated an inclination to the younger age groups.

Average proportion of years of potential life lost in working age (YPLL in WA) due to major cardiovascular diseases among the population under 70 years in Republic of Moldova between 2008-2012 was $70 \%$ (respectively, ischemic heart disease $74.5 \%$, cerebrovascular disease $-74,4 \%$, hypertensive disease - 61\%). Mortality causes calculated using YPLL statistics differ from those obtained through other methods: reflects the cause of mortality, which influences economically active 
young population and determines the biggest economic losses.

Average number of YPLL for each occurred death due to hypertensive decease demonstrated a stable decrease in the investigation period from 8,4 YPLL in year 2008 down to 7,5 YPLL in year 2012, which indicates a stable inclination towards superior age groups (YPLL influence is less).

While, cerebrovascular disease shown a set YPLL value of 10,1 in the period 20082012, which indicates a persistence of premature decease phenomenon among younger population (maintaining the same quality characteristics of age).

Ischemic heart disease shown values that represent an area with low increase tendency from 10,3 YPLL for each produced death in 2008 and 10,5 YPLL for each produced death in 2012. This indicates an inclination towards age groups with a more important YPLL contribution younger.

Years of Potential Life Lost represents a quantitative indicator of premature mortality, reflects mortalities tendencies of young age groups by not only considering deaths' medical causes but also age groups' (age when death occurred).

Obtained results showed that using statistical data focused on loss (YPLL) and not on occurrence of phenomenon allows to determine disadvantage population groups, intervention priorities for health services, better identification of disadvantaged population groups, determining the most optimal criterions for health resources allocation, determining priorities for health research.

Premature deaths can be avoided if community action and prevention measures would be applied correctly and on time. Generally, a higher importance is shown towards medical care and less to the preventive aspect, forgetting the idea that an efficient prophylaxis can substantially reduce premature mortality and can add a substantial number to years of life expectancy.

\section{Conclusions}

1. Ensuring a systematic monitoring and evaluation of health status using potential statistic indicators (YPLL) in the Republic of Moldova will contribute in prioritization in age group and individual interventions.

2. By using YPLL for systematic elaboration of complex indicators, which at the same time will take into account phenomenon of premature mortality intensity and non-fatal consequences of causes of illness - disability adjusted life years (DALY), will contribute qualitatively in evaluation of cardiovascular disease burden and risk management efficiency in the Republic of Moldova.

3. Systematic evaluation of potential statistical indicators (YPLL, DALY) will ensure an additional insurance when making decisions based on the evidence of cardiovascular risk management in the Republic of Moldova.

\section{References}

1. Global burden of disease 2004 update. Available from: http://www.who.int/healthinfo/global burden di sease/GBD report 2004update full.pdf

$2 . \quad$ World Health Organization (WHO). Global Atlas on cardiovascular disease prevention and control. World Health Organization (2011) Available from: http://www.who.int/cardiovascular_diseases/pub lications/atlas_cvd/en/

3. Programul Național de prevenire și control al bolior cardiovasculare pentru anii 2014-2020. Hotărîrea Guvernului nr.300 din 20 aprilie 2014. Monitorul Oficial (06.05.2014) Nr.104-109 art. Nr:327

$4 . \quad$ World Health Organization (WHO). Global status report on noncommunicable diseases 2010. World Health Organization (2011).

http://www.who.int/nmh/publications/ncd_repor t2010/en/

5. World Health Organization (WHO). Global Action Plan for the Prevention and Control of NCDs 2013-2020. WHO (2013):http://www.who.int/nmh/events/ncd_acti on_plan/en/

6. Centrul Naţional de Management în Sănătate: http://www.cnms.md/ro/rapoarte 